\title{
Comparative beneficiary effects of immunotherapy against chemotherapy in patients with advanced NSCLC: Meta-analysis and systematic review
}

\author{
DA-PING YU, XU CHENG, ZHI-DONG LIU and SHAO-FA XU
}

Department of Thoracic Surgery, Beijing Chest Hospital, Capital Medical University, Beijing 101149, P.R. China

Received February 7, 2017; Accepted May 4, 2017

DOI: $10.3892 / \mathrm{ol} .2017 .6274$

\begin{abstract}
Lung cancer is the most commonly diagnosed cancer among men and it is the third ranked in women. There are two major types of lung cancer, namely, small cell lung cancer (SCLC), which accounts for $\sim 20 \%$ of the cases, and non-small cell lung cancer (NSCLC), which is the most common. Chemotherapy and chemoradiotherapy have been used as the first-line therapies but suffer from lack of efficacy and also of several toxic adverse effects. Immunotherapeutic approaches including tumor antigen vaccination, monoclonal antibodies targeting checkpoint pathways and also activated immune cells are being developed and have been shown to be effective in treating NSCLC. Despite their promise, efficacy of several immunotherapies has not been consistent. We undertook this meta-analysis study to analyze results from clinical trials that compared efficacy and safety of immunotherapies with placebo or chemotherapy/radiotherapy in improving overall survival (OS) and progression-free survival (PFS) of NSCLC patients. Various databases were searched to identify randomized clinical studies examining the efficacy and safety of antibody- and vaccine-based immunotherapies in NSCLC patients in comparison to chemotherapy or chemoradiotherapy or placebo. Effects on OS and PFS and also adverse events have been compared. In accordance with the selection criteria, a total of 13 studies with 3,513 patients in immunotherapy and 3,072 patients in chemotherapy/placebo, were selected. PFS (odds ratio 1.81, 95\% CI 1.36, 2.42; $\mathrm{P}<0.0001)$ and OS $(\mathrm{P}<0.0001)$ are found to be greatly improved by immunotherapies. Immunotherapy of NSCLC patients was also found to prevent several adverse effects and to improve daily living ability of the patients. The present meta-analysis strongly suggests that immunotherapy improves OS and PFS of patients with NSCLC.
\end{abstract}

Correspondence to: Dr Shao-Fa Xu, Department of Thoracic Surgery, Beijing Chest Hospital, Capital Medical University, 97 Machang Road, Tongzhou, Beijing 101149, P.R. China E-mail: xm32qf@163.com

Key words: chemotherapy, lung cancer, non-small cell lung cancer, immunotherapy, vaccine therapy, antibody therapy, immune checkpoint inhibitors, progression-free survival, overall survival

\section{Introduction}

According to worldwide cancer statistics (Globocan 2012), lung cancer is the most commonly diagnosed cancer among men, and in women it is the third most commonly diagnosed (1). Approximately 2 million new cases of lung cancer and 1.5 million lung cancer related deaths are recorded each year (2). Mortality due to lung cancer is estimated to increase up to 10 million per year in another 15 years (3). There are two major types of lung cancer, viz., small cell lung cancer (SCLC), which is less common accounting for $20 \%$ of the cancer cases, and non-small cell lung cancer (NSCLC), which is the most common, accounting for almost $85 \%$ of lung cancer cases (4). Despite the high incidence and associated mortality, the available treatment options and prognosis for NSCLC are not satisfactory. In a recent meta-analysis, we showed that exercise at medium to high level intensity is associated with lower risk of lung cancer, both in men and women, indicating the lifestyle-based benefits for reducing lung cancer risk (5). Platinum-doublet chemotherapy, which is the standard first-line therapy, has been shown to yield objective responses with a median overall survival (OS) of 8-10 months in approximately $30-40 \%$ of patients with NSCLC and there are significant safety issues (6). Considering that the efficacy of platinum-based therapies is dependent on the mutational/single nucleotide polymorphisms (SNP) status of the components of DNA-damage repair systems available in the target cancer cells, there can be significant individual differences in treatment responses. Thus, we showed earlier that the SNPs XRCC1Arg399GIn and XPG His46His are associated with significantly better treatment response in NSCLC patients to platinum-based chemotherapy (7). On the other hand, tyrosine kinase inhibitors (e.g., gefitinib, erlotinib, and afatinib) are found to be more effective with nearly $65 \%$ response rate and a median OS of 24-36 months in NCLSC patients with EGFR mutations (8). In addition to chemotherapy and chemoradiotherapy, immunological approaches have been shown to be effective in treating various cancers, including NSCLC. Significant advances have been made in the last 3 years in the development of immunotherapies for NSCLC. Immunotherapies include tumor antigen vaccination, monoclonal antibodies targeting checkpoint pathways and also activated immune cells (8-10). Several recent studies have shown the efficacy of immunotherapies against advanced 
stage NSCLC patients (10-12). Despite the promise of several immunotherapies, their efficacy has not been consistent as noted in the phase III FORTIS trial (13). Response to immunotherapies can also be dependent on many other factors, such as chemokines that influence immune cell function. Thus, we reported earlier that polymorphism $(-2518 \mathrm{~A} / \mathrm{G})$ in the gene coding for monocyte chemoattractant protein-1 (MCP-1) is associated with elevated risk for NSCLC. MCP-1 is known to be a tumor suppressor via pathways involving T-lymphocytes or independent of lymphocytes (14). Similarly, we also noted that the frequencies of polymorphisms in the human leukocyte antigen (HLA) system, which is involved in the regulation of immune response, HLA-A*0201, A*2601, B*1518, B*3802, DRB1*0401, DRB1*0402, and DRB1*1201 are higher in the lung cancer patients than healthy controls (15), and affect the tumor immunity.

An earlier meta-analysis of 12 randomized controlled trials of immunotherapies indicated a beneficial effect on OS with few adverse effects (6). However, in this analysis, 3 monoclonal antibody (Mab)-based trials with cetuximab (targets EGFR) and one trial with trastuzumab (targets HER2) as NSCLC Mab therapy subgroup, whereas these actually are related to the growth factors and not immune system. A recent meta-analysis of several clinical trials of immunotherapies, including therapeutic vaccines and immune checkpoint inhibitors showed that immunotherapies are well tolerated in advanced NSLSC patients and improve OS and that specific antitumor immune response simulating agents are more efficacious than immunomodulatory type agents (16). A comparison of tumor vaccine-based therapies with cellular immunotherapies, in a meta-analysis revealed that cellular immunotherapies are more effective in improving OS and progression-free survival (PFS) in NSCLC patients (12). However, in many of these meta-analyses, the number of included studies and the total number of patients was lower and also, as mentioned above, inclusion of unrelated therapies could have complicated the outcomes and conclusions. Also, discordant results were seen in certain clinical trials using immunotherapies against NSCLC as the promising phase II study results were not observed in phase III study, leading to premature termination of the clinical trials, questioning the efficacy of immunotherapy (17). We have now conducted a meta-analysis of 13 clinical trials assessing the efficacy and safety of Mab therapies directed against tumor antigens and tumor antigen-based vaccination therapies, as compared to chemotherapy/chemoradiotherapy in NSCLC patients.

In the present meta-analysis, we analyzed results from clinical trial studies that simultaneously compared immunotherapies with placebo or chemotherapy/radiotherapy to assess the beneficial effects of immunotherapies collectively or separately in improving OS and PFS of NSCLC patients. Besides efficacy, we also examined if immunotherapies are better in terms of safety, by looking at the treatment-related adverse effects.

\section{Materials and methods}

Criteria for considering studies for this review. Randomized controlled phase II and III clinical trials on patients with NSCLC at stage III and IV, with histological confirmation are included in this analysis. In these studies, immunotherapies were administered to patients along with chemotherapy or as monotherapy and the treatment effects were compared to a control group of patients, who received either chemotherapy alone or placebo. For the present meta-analysis, we have included only vaccine-based and Mab-based immunotherapy studies and not the immune cell-based adoptive immunotherapy clinical trials. Studies that did not include proper controls were excluded from this meta-analysis. Most of the patients in the included studies had Eastern Cooperative Oncology Group (ECOG) performance status of 0,1 or 2 (18). Patients were excluded if they were on concurrent systemic steroids, with metastases in bone requiring immediate therapy, uncontrolled pleural effusions, serious non-malignant disease or previous malignancies or if they had myocardial infarction or other cardiovascular disease in the 6-month period prior to study. Approximately half of the included studies were open-label, whereas the remaining are double-blind studies.

Search methods. Literature search was completed on 10 January 2017 and publications that included complete relevant information and data were collected. Following databases were searched: PubMed, Google Scholar, Scopus, Web of Science and Cochrane Central Register. Search MeSH terms included lung cancer, NSCLC, immunotherapy, vaccine therapy for NSCLC, vaccination for NSCLC, OS, clinical trials, PFS and Mab therapy. All the relevant publications only in English language were collected and were initially screened at the title and abstract level. Only clinical trials at phase II and III level were included in this analysis. Full reports and supplemental information files were retrieved as per the relevance of the selected study.

Data collection and analysis and quality assessment. All the authors of this meta-analysis participated in the screening of collected publications and data extraction. Data including patient baseline characteristics such as age, sex, description and dosages of the administered treatment, tumor histology, and disease stage, and treatment primary endpoint measurements (PFS events, OS) and treatment related adverse effects were collected, by filling out a pre-defined data collection form, from each included study. OS, which was defined as the time from randomization to censor or death due to any cause, was mostly reported as median months and for the purposes of meta-analysis, these values were converted to mean \pm SD. The period from randomization of patients to the progression of disease (or death if it occurred first), during the study duration, as ascertained and documented by the study investigators, was defined as PFS. Treatment related adverse effects were evaluated to assess the safety of immunotherapy procedure, and the adverse events (scoring grade $\geq 3$ ) reported in more than two clinical trials, as the number of patients were collected. These include both hematological and non-hematological effects. Hematological events recorded were anemia, neutropenia, thrombocytopenia and the non-hematological events include fatigue, vomiting, abdominal pain, fever, nausea, and anorexia/loss of appetite.

Statistical analysis. Statistical analyses were conducted using the methods described by the Cochrane Collaboration guidelines for meta-analysis, using Review Manager (RevMan) 
version 5.3 software (the Cochrane Collaboration). As mentioned above, OS was mostly reported as median months and lower and upper limits of the range and for the purposes of meta-analysis, these values were converted to mean \pm SD. Number of PFS events, were not given directly, was deduced from the PFS plots in the individual trials. The effect of immunotherapy on PFS and each of the adverse events were analyzed by Mantel-Haenszel statistics, odds ratios (OR) in the random-effect model, at 95\% confidence intervals (CI). The effect of immunotherapy on OS was assessed by mean difference analyses in inverse variance (IV) fixed mode at 95\% confidence intervals. Sensitivity analysis was performed by excluding one study at a time and also by removing study with highest weightage, among the included data to examine influence of bias on the deduced statistical significance and interpretation. Risk ratios were calculated for adverse events and also for PFS at $95 \%$ confidence intervals.

\section{Results}

Search from all the databases yielded 12,338 studies, which contained the search MeSH terms either in the title or in the abstract. Exclusion of studies was done through the participation of all authors, who read the abstracts and a collective decision was adopted. A total of 13 studies $(11,19-29)$ were identified on the basis of relevancy and inclusion/exclusion criteria and completeness of the data in the study (Fig. 1). As immunotherapy of NSCLC is a recently developed treatment approach, all the studies identified are published after 2011. The total number of patients in all the included studies was 6,585 and the number of patients who received immunotherapy was 3,513 , whereas the control group patients who received chemotherapy or placebo were 3,072 (Fig. 1).

Patient characteristics. The demographic and baseline characteristics of patients in the included studies are given in Table I. Average age of the patients in both immunotherapy and control groups was $\sim 62$ years. Nearly $64.3 \%$ of the patients who received immunotherapy were males while in control chemotherapy group $62.9 \%$ were males. Tumors were of adenocarcinoma type, by histological examination in nearly two-thirds of the patients in both immunotherapy group $(n=2,155)$ and in control group $(n=2,009)$ and next major histological type was squamous cell carcinoma (Table I). ECOG performance status, which is an indicator of the patient's daily living ability and the progress of disease, is predominantly 0 or 1 for most of the included patients, in both immunotherapy and chemotherapy/placebo groups (18). Majority of the patients are restricted for strenuous physical activity, even though they could carry out light and sedentary work.

In the present meta-analysis study, only antibody-based and vaccine-based immunotherapies are included. Cellular therapy studies were not included as many of these studies are at early phase I stage. Out of the 13 studies, nine were antibody-based immunotherapies, while the remaining four were vaccine-based. Antibody therapies included ipilimumab, an anti-cytotoxic T-cell lymphocyte-4 Mab (20); nivolumab, a human IgG4 PD-1 immune-checkpoint-inhibitor Mab (11,21); atezolizumab, another anti-PD-L1 Mab $(22,26)$; pembrolizumab, also known as MK-3475, a human IgG4 PD-1

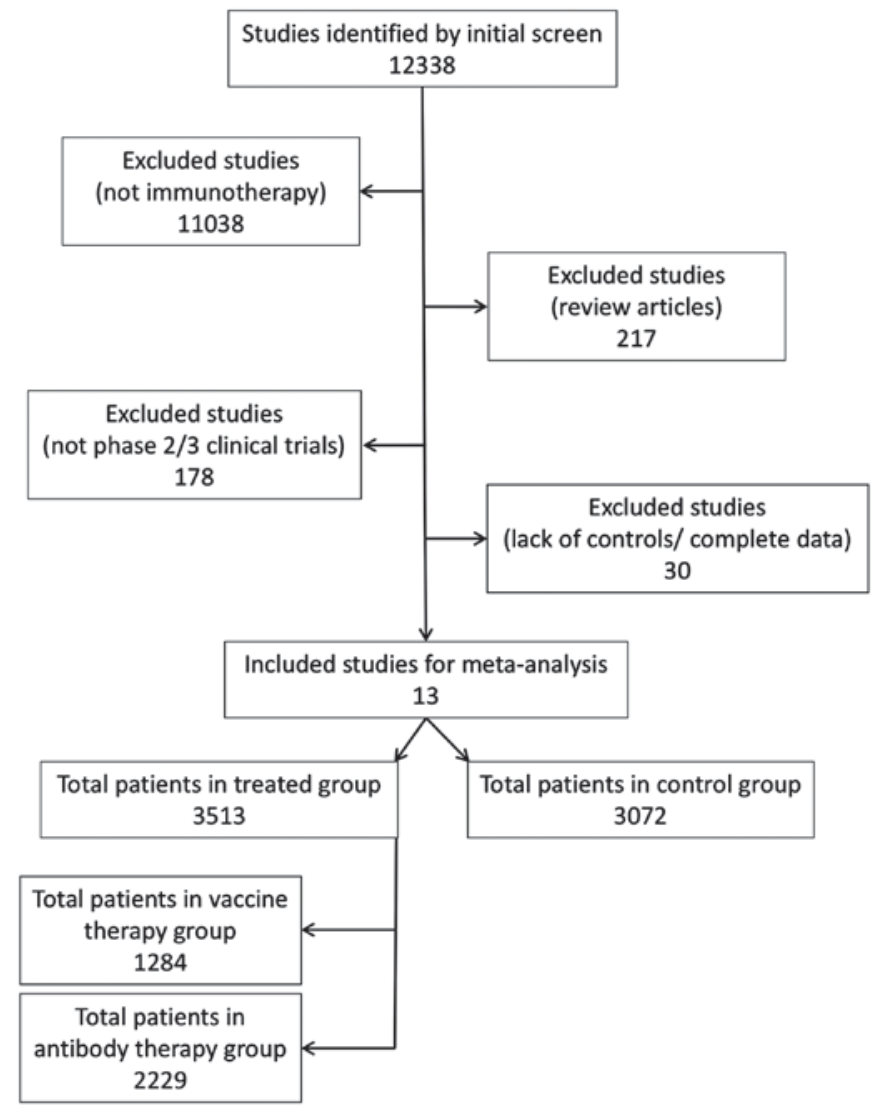

Figure 1. Flow chart of selection of studies for the meta-analysis.

Mab $(23,25)$; and bevacizumab, a recombinant, anti-vascular endothelial growth factor Mab (28). Vaccine-based immunotherapies included TG4010 genetic vaccine, which is a suspension of a recombinant modified vaccinia virus strain Ankara (MVA), coding for the MUC1 tumor-associated antigen and interleukin-2 (19,24); tecemotide (L-BLP25), a tumor associated MUC1 antigen-specific immunotherapy, that is capable of inducing a T-cell response to MUC1 (27) and belagenpumatucel-L, an allogeneic whole tumor cell vaccine consisting of four pCHEK/HBA2 (human transforming growth factor- $\beta 2$-antisense vector)-transfected NSCLC cell lines (29). Immunotherapy dosages and chemotherapy dosages are shown in Table I. Dosage of antibodies varied depending on the study and antibody used. Thus, ipilimumab was given at $10 \mathrm{mg} / \mathrm{kg}$ as four doses plus paclitaxel and carboplatin followed by two doses of placebo plus paclitaxel and carboplatin, intravenously every 3 weeks for up to 18 weeks (20). Nivolumab was given at $3 \mathrm{mg} / \mathrm{kg}$, every 2 weeks, for 11 to 13.2 months $(11,21)$. Atezolizumab was given at $1.2 \mathrm{~g}$ fixed dose, intravenously on day 1 , every 3 weeks, for at least 9 months $(22,26)$. Pembrolizumab was given at $2 \mathrm{mg}$ or $10 \mathrm{mg} / \mathrm{kg}$, every 3 weeks for 24 months (23) or at $200 \mathrm{mg}$ every 3 weeks in 35 cycles (25). Bevacizumab was given at $15 \mathrm{mg} / \mathrm{kg}$, intravenously on day 1 , every 3 weeks + erlotinib $150 \mathrm{mg} / \mathrm{day}$ (28). TG4010 genetic vaccine was administered at $10^{8} \mathrm{pfu}$ along with cisplatin $75 \mathrm{mg} / \mathrm{m}^{2}$ on day 1 ; gemcitabine $1.25 \mathrm{~g} / \mathrm{m}^{2}$ on day 1 and 8 , every 3 weeks in 6 cycles $(19,24)$. Tecemotide was administered subcutaneously, as $806 \mu \mathrm{g}$ lipopeptide with lipid-A and liposome forming 
Table I. Baseline characteristics of patients included in the meta-analysis.

A, Immunotherapy

\begin{tabular}{|c|c|c|c|c|c|c|c|c|c|c|c|}
\hline \multirow{2}{*}{$\begin{array}{l}\text { Author } \\
\text { (ref.) year }\end{array}$} & \multirow[b]{2}{*}{ Study type } & \multirow{2}{*}{$\begin{array}{c}\text { Type of } \\
\text { immunotherapy }\end{array}$} & \multirow{2}{*}{$\begin{array}{l}\text { No. of } \\
\text { patients }\end{array}$} & \multirow{2}{*}{$\begin{array}{l}\text { Age, } \\
\text { years }\end{array}$} & \multirow{2}{*}{$\begin{array}{c}\text { Males, } \\
\%\end{array}$} & \multirow[b]{2}{*}{ Immunotherapy dosage } & \multicolumn{3}{|c|}{$\begin{array}{l}\text { ECOG } \\
\text { status (no. } \\
\text { of patients) }\end{array}$} & \multicolumn{2}{|c|}{$\begin{array}{l}\text { Histology } \\
\text { type (n) }\end{array}$} \\
\hline & & & & & & & 0 & 1 & 2 & Ad & $\mathrm{Sq}$ \\
\hline $\begin{array}{l}\text { Quoix } \\
\text { et al (19) } \\
2011\end{array}$ & $\begin{array}{l}\text { Controlled } \\
\text { multicenter } \\
\text { phase 2B }\end{array}$ & $\begin{array}{l}\text { TG4010 genetic } \\
\text { vaccine (targets } \\
\text { tumor MUC1) }\end{array}$ & 74 & 58.5 & 71.6 & $\begin{array}{l}10^{8} \mathrm{pfu} ; \text { cisp } 75 \mathrm{mg} / \mathrm{m}^{2} \\
\text { on day } 1 ; \text { gem } 1.25 \mathrm{~g} / \mathrm{m}^{2} \\
\text { on day } 1,8 ; \text { every } \\
3 \text { weeks x } 6 \text { cycles }\end{array}$ & 20 & 53 & 1 & 47 & 19 \\
\hline $\begin{array}{l}\text { Lynch } \\
\text { et al (20) } \\
2012\end{array}$ & $\begin{array}{l}\text { Randomized, } \\
\text { double-blind } \\
\text { phase 2B }\end{array}$ & $\begin{array}{l}\text { Ipilimumab (anti- } \\
\text { cytotoxic T-cell } \\
\text { lymphocyte-4 Mab) }\end{array}$ & 70 & 59 & 76 & $\begin{array}{l}10 \mathrm{mg} / \mathrm{kg} \text { Ipilimumab; } \\
\text { pacli } 175 \mathrm{mg} / \mathrm{m}^{2} \text {; carbo } \\
\text { AUC } 6 \text {; i.v. dosing } \\
\text { every } 3 \text { weeks x } 6 \text { cycles }\end{array}$ & 19 & 51 & & 35 & 21 \\
\hline $\begin{array}{l}\text { Borghaei } \\
\text { et al (21) } \\
2015\end{array}$ & $\begin{array}{l}\text { Randomized, } \\
\text { open-label } \\
\text { phase } 3\end{array}$ & $\begin{array}{l}\text { Nivolumab } \\
\text { human IgG4 PD-1 } \\
\text { immune checkpoint } \\
\text { inhibitor Mab }\end{array}$ & 292 & 61 & 52 & $\begin{array}{l}3 \mathrm{mg} / \mathrm{kg} \text {, every } 2 \text { weeks; } \\
\text { for } 13.2 \text { months } \\
\text { minimum }\end{array}$ & 84 & 208 & & 268 & 7 \\
\hline $\begin{array}{l}\text { Brahmer } \\
\text { et al (11) } \\
2015\end{array}$ & $\begin{array}{l}\text { Randomized, } \\
\text { open-label, } \\
\text { multicenter, } \\
\text { phase } 3\end{array}$ & $\begin{array}{l}\text { Nivolumab } \\
\text { (IgG4 PD-1 Mab) }\end{array}$ & 135 & 62 & 82 & $3 \mathrm{mg} / \mathrm{kg}$, every 2 weeks & 27 & 106 & & & \\
\hline $\begin{array}{l}\text { Fehrenbacher } \\
\text { et al (22) } \\
2016\end{array}$ & $\begin{array}{l}\text { Multicenter, } \\
\text { open-label, } \\
\text { randomized } \\
\text { phase } 2\end{array}$ & $\begin{array}{l}\text { Atezolizumab } \\
\text { (anti-PD-L1 Mab ) }\end{array}$ & 144 & 62 & 65 & $\begin{array}{l}\text { Atezolizumab } 1.2 \mathrm{~g} \\
\text { fixed, i.v., on day } 1 \text {, } \\
\text { every } 3 \text { weeks }\end{array}$ & 46 & 96 & & 95 & 49 \\
\hline $\begin{array}{l}\text { Herbst } \\
\text { et al (23) } \\
2016\end{array}$ & $\begin{array}{l}\text { Multicenter, } \\
\text { randomized, } \\
\text { open-label, } \\
\text { phase } 2 / 3\end{array}$ & $\begin{array}{l}\text { Pembrolizumab } \\
\text { (MK-3475; human } \\
\text { IgG4 PD-1 Mab) }\end{array}$ & 344 & 63 & 62 & $\begin{array}{l}\text { Pembrolizumab, } \\
2 \mathrm{mg} / \mathrm{kg} \text {, every } \\
3 \text { weeks }\end{array}$ & 112 & 229 & 3 & 240 & 76 \\
\hline $\begin{array}{l}\text { Herbst } \\
\text { et al (23) } \\
2016\end{array}$ & $\begin{array}{l}\text { Multicenter, } \\
\text { randomized, } \\
\text { open-label, } \\
\text { phase } 2 / 3\end{array}$ & $\begin{array}{l}\text { Pembrolizumab } \\
\text { (MK-3475; human } \\
\text { IgG4 PD-1 Mab) }\end{array}$ & 346 & 63 & 62 & $\begin{array}{l}\text { Pembrolizumab, } \\
10 \mathrm{mg} / \mathrm{kg} \text {, } \\
\text { every } 3 \text { weeks }\end{array}$ & 120 & 225 & 1 & 244 & 80 \\
\hline $\begin{array}{l}\text { Quoix } \\
\text { et al (24) } \\
2016\end{array}$ & $\begin{array}{l}\text { Randomized, } \\
\text { double-blind } \\
\text { multicenter, } \\
\text { controlled } \\
\text { phase } 2 \mathrm{~b} / 3\end{array}$ & $\begin{array}{l}\text { TG4010 } \\
\text { genetic vaccine }\end{array}$ & 111 & 63 & 65 & $\begin{array}{l}10^{8} \mathrm{pfu} \text {; every week for } 6 \\
\text { weeks then every } 3 \text { weeks; } \\
\text { cisp } 75 \mathrm{mg} / \mathrm{m}^{2} \text { on day } 1 ; \\
\text { gem } 1.25 \mathrm{~g} / \mathrm{m}^{2} \text { on day } 1,8\end{array}$ & 33 & 77 & & 95 & 13 \\
\hline $\begin{array}{l}\text { Reck } \\
\text { et al (25) } \\
2016\end{array}$ & $\begin{array}{l}\text { Open-label, } \\
\text { multicenter, } \\
\text { phase } 3\end{array}$ & $\begin{array}{l}\text { Pembrolizumab } \\
\text { (MK-3475; human } \\
\text { IgG4 PD-1 Mab) }\end{array}$ & 154 & 64.5 & 59.7 & $\begin{array}{l}\text { Pembrolizumab, } \\
200 \text { mg every } 3 \text { weeks, } \\
35 \text { cycles }\end{array}$ & 54 & 99 & & 125 & 29 \\
\hline $\begin{array}{l}\text { Rittmeyer } \\
\text { et al (26) } \\
2017\end{array}$ & $\begin{array}{l}\text { Open-label, } \\
\text { multicenter } \\
\text { randomized } \\
\text { controlled } \\
\text { phase } 3\end{array}$ & $\begin{array}{l}\text { Atezolizumab } \\
\text { (Anti-PD-L1 Mab) }\end{array}$ & 425 & 63 & 61 & $\begin{array}{l}\text { atezolizumab } 1.2 \mathrm{~g} \\
\text { fixed, i.v., on day } 1 \text {, } \\
\text { every } 3 \text { weeks }\end{array}$ & 155 & 270 & & 313 & 112 \\
\hline $\begin{array}{l}\text { Butts } \\
\text { etal (27) } \\
2014\end{array}$ & $\begin{array}{l}\text { START } \\
\text { randomized, } \\
\text { double-blind, } \\
\text { multicenter phase } 3\end{array}$ & $\begin{array}{l}\text { Tecemotide } \\
\text { vaccine (MUC1- } \\
\text { antigen-specific } \\
\text { immunotherapy) }\end{array}$ & 829 & 61 & 68 & $\begin{array}{l}\text { Subcutaneous } \\
\text { tecemotide }(806 \mu \mathrm{g} \\
\text { lipopeptide })+ \text { lipid-A+ } \\
\text { liposome forming lipids }\end{array}$ & 398 & 427 & & 289 & 401 \\
\hline
\end{tabular}


Table I. Continued.

A, Immunotherapy

\begin{tabular}{|c|c|c|c|c|c|c|c|c|c|c|c|}
\hline \multirow{2}{*}{$\begin{array}{l}\text { Author } \\
\text { (ref.) year }\end{array}$} & \multirow[b]{2}{*}{ Study type } & \multirow{2}{*}{$\begin{array}{c}\text { Type of } \\
\text { immunotherapy }\end{array}$} & \multirow{2}{*}{$\begin{array}{l}\text { No. of } \\
\text { patients }\end{array}$} & \multirow{2}{*}{$\begin{array}{l}\text { Age, } \\
\text { years }\end{array}$} & \multirow{2}{*}{$\begin{array}{l}\text { Males, } \\
\%\end{array}$} & \multirow{2}{*}{ Immunotherapy dosage } & \multicolumn{3}{|c|}{$\begin{array}{l}\text { ECOG } \\
\text { status (no. } \\
\text { of patients) }\end{array}$} & \multicolumn{2}{|c|}{$\begin{array}{l}\text { Histology } \\
\text { type (n) }\end{array}$} \\
\hline & & & & & & & 0 & 1 & 2 & Ad & $\mathrm{Sq}$ \\
\hline $\begin{array}{l}\text { Herbst } \\
\text { et al (28) } \\
2011\end{array}$ & $\begin{array}{l}\text { Double-blind, } \\
\text { multicenter } \\
\text { placebo-controlled } \\
\text { phase } 3\end{array}$ & $\begin{array}{l}\text { Bevacizumab } \\
\text { (recombinant, anti- } \\
\text { vascular endothelial } \\
\text { growth factor Mab) }\end{array}$ & 319 & 65 & 54 & $\begin{array}{l}\text { Bevacizumab at } \\
15 \mathrm{mg} / \mathrm{kg}, \mathrm{i} . \mathrm{v} ., \text { on day } \\
1, \text { every } 3 \text { weeks + } \\
\text { erlotinib } 150 \mathrm{mg} / \text { day }\end{array}$ & 129 & 166 & 23 & 242 & 23 \\
\hline $\begin{array}{l}\text { Giaccone } \\
\text { et al (29) } \\
2015\end{array}$ & Phase 3 study & $\begin{array}{l}\text { Belagenpumatucel-L } \\
\text { (whole tumor cell } \\
\text { vaccine, of NSCLC) } \\
\text { cells, transfected with } \\
\text { a human TGF- } \beta 2- \\
\text { antisense vector }\end{array}$ & 270 & 61.5 & 58 & $\begin{array}{l}2.5 \times 10^{7} \text { total cells } \\
\text { were injected } \\
\text { intradermally }\end{array}$ & 119 & 139 & 7 & 162 & 65 \\
\hline
\end{tabular}

B, Chemotherapy/placebo

\begin{tabular}{|c|c|c|c|c|c|c|c|c|c|c|c|}
\hline \multirow{2}{*}{$\begin{array}{l}\text { Author } \\
\text { (ref.) year }\end{array}$} & \multirow[b]{2}{*}{ Study type } & \multirow{2}{*}{$\begin{array}{c}\text { Type of } \\
\text { chemotherapy }\end{array}$} & \multirow{2}{*}{$\begin{array}{l}\text { No. of } \\
\text { patients }\end{array}$} & \multirow{2}{*}{$\begin{array}{l}\text { Age, } \\
\text { years }\end{array}$} & \multirow{2}{*}{$\begin{array}{l}\text { Males, } \\
\%\end{array}$} & \multirow[b]{2}{*}{ Chemotherapy dosage } & \multicolumn{3}{|c|}{$\begin{array}{l}\text { ECOG } \\
\text { status (no. } \\
\text { of patients) }\end{array}$} & \multicolumn{2}{|c|}{$\begin{array}{l}\text { Histology } \\
\text { type (n) }\end{array}$} \\
\hline & & & & & & & 0 & 1 & 2 & Ad & $\mathrm{Sq}$ \\
\hline $\begin{array}{l}\text { Quoix } \\
\text { et al (19) } \\
2011\end{array}$ & $\begin{array}{l}\text { Controlled } \\
\text { multicenter } \\
\text { phase 2B }\end{array}$ & $\begin{array}{l}\text { Cisplatin + } \\
\text { gemcitabine }\end{array}$ & 74 & 58.5 & 73 & $\begin{array}{l}\text { Cisp } 75 \mathrm{mg} / \mathrm{m}^{2} \text { on } \\
\text { day } 1 ; \text { gem } 1.25 \mathrm{~g} / \mathrm{m}^{2} \\
\text { on day } 1,8 ; \text { every } \\
3 \text { weeks x } 6 \text { cycles }\end{array}$ & 20 & 54 & 0 & 55 & 11 \\
\hline $\begin{array}{l}\text { Lynch } \\
\text { et al (20) } \\
2012\end{array}$ & $\begin{array}{l}\text { Randomized } \\
\text { double-blind } \\
\text { phase 2B }\end{array}$ & $\begin{array}{l}\text { Paclitaxel + } \\
\text { carboplatin }\end{array}$ & 66 & 62 & 74 & $\begin{array}{l}\text { Pacli } 175 \mathrm{mg} / \mathrm{m}^{2} ; \\
\text { carbo AUC 6; i.v. } \\
\text { dosing every } 3 \text { weeks } \\
\text { x } 6 \text { cycles }\end{array}$ & 15 & 51 & & 38 & 15 \\
\hline $\begin{array}{l}\text { Borghaei } \\
\text { et al (21) } \\
2015\end{array}$ & $\begin{array}{l}\text { Randomized } \\
\text { open-label, } \\
\text { phase } 3\end{array}$ & Docetaxel & 290 & 64 & 58 & $\begin{array}{l}\text { Docetaxel } 75 \mathrm{mg} / \mathrm{m}^{2} \\
\text { every } 3 \text { weeks; for } \\
13.2 \text { months minimum }\end{array}$ & 95 & 194 & & 273 & 7 \\
\hline $\begin{array}{l}\text { Brahmer } \\
\text { et al }(11) \\
2015\end{array}$ & $\begin{array}{l}\text { Randomized } \\
\text { open-label, } \\
\text { multicenter } \\
\text { phase } 3\end{array}$ & Docetaxel & 137 & 64 & 71 & $\begin{array}{l}\text { Docetaxel } 75 \mathrm{mg} / \mathrm{m}^{2} \\
\text { every } 3 \text { weeks }\end{array}$ & 37 & 100 & & & \\
\hline $\begin{array}{l}\text { Fehrenbacher } \\
\text { et al }(22) \\
2016\end{array}$ & $\begin{array}{l}\text { Multicenter, } \\
\text { open-label, } \\
\text { randomized } \\
\text { phase } 2\end{array}$ & Docetaxel & 143 & 62 & 53 & $\begin{array}{l}\text { Docetaxel } 75 \mathrm{mg} / \mathrm{m}^{2} \\
\text { on day } 1 \text {, every } \\
3 \text { weeks }\end{array}$ & 45 & 97 & & 95 & 48 \\
\hline $\begin{array}{l}\text { Herbst } \\
\text { et al (23) } \\
2016\end{array}$ & $\begin{array}{l}\text { Multicenter, } \\
\text { randomized, } \\
\text { open-label, } \\
\text { phase } 2 / 3\end{array}$ & Docetaxel & 343 & 62 & 61 & $\begin{array}{l}\text { Docetaxel } 75 \mathrm{mg} / \mathrm{m}^{2} \\
\text { on day } 1 \text {, every } \\
3 \text { weeks }\end{array}$ & 116 & 224 & 1 & 240 & 66 \\
\hline $\begin{array}{l}\text { Quoix } \\
\text { et al (24) } \\
2016\end{array}$ & $\begin{array}{l}\text { Randomized, } \\
\text { double-blind } \\
\text { multicenter, }\end{array}$ & $\begin{array}{l}\text { Cisplatin + } \\
\text { gemcitabine }\end{array}$ & 111 & 59 & 63 & $\begin{array}{l}\text { Cisp } 75 \mathrm{mg} / \mathrm{m}^{2} \text { on } \\
\text { day } 1 ; \text { gem } 1.25 \mathrm{~g} / \mathrm{m}^{2} \\
1, \text { every } 3 \text { weeks }+\end{array}$ & 35 & 76 & & 90 & 13 \\
\hline
\end{tabular}


Table I. Continued.

B, Chemotherapy/placebo

\begin{tabular}{|c|c|c|c|c|c|c|c|c|c|c|c|}
\hline \multirow{2}{*}{$\begin{array}{l}\text { Author } \\
\text { (ref.) year }\end{array}$} & \multirow[b]{2}{*}{ Study type } & \multirow{2}{*}{$\begin{array}{c}\text { Type of } \\
\text { chemotherapy }\end{array}$} & \multirow{2}{*}{$\begin{array}{l}\text { No. of } \\
\text { patients }\end{array}$} & \multirow{2}{*}{$\begin{array}{l}\text { Age, } \\
\text { years }\end{array}$} & \multirow{2}{*}{$\begin{array}{l}\text { Males, } \\
\%\end{array}$} & \multirow[b]{2}{*}{ Chemotherapy dosage } & \multicolumn{3}{|c|}{$\begin{array}{l}\text { ECOG } \\
\text { status (no. } \\
\text { of patients) }\end{array}$} & \multicolumn{2}{|c|}{$\begin{array}{l}\text { Histology } \\
\text { type (n) }\end{array}$} \\
\hline & & & & & & & 0 & 1 & 2 & Ad & $\mathrm{Sq}$ \\
\hline & $\begin{array}{l}\text { controlled } \\
\text { phase } 2 b / 3\end{array}$ & & & & & $\begin{array}{l}\text { on day } 1,8 ; \text { every } \\
3 \text { weeks x } 6 \text { cycles }\end{array}$ & & & & & \\
\hline $\begin{array}{l}\text { Reck } \\
\text { et al (25) } \\
2016\end{array}$ & $\begin{array}{l}\text { Open-label, } \\
\text { multicenter, } \\
\text { phase } 3\end{array}$ & $\begin{array}{l}\text { Carboplatin/ } \\
\text { cisplatin/paclitaxel/ } \\
\text { gemcitabine }\end{array}$ & 151 & 66 & 62.9 & Varying dosages & 53 & 98 & & 124 & 27 \\
\hline $\begin{array}{l}\text { Rittmeyer } \\
\text { et al (26) } \\
2017\end{array}$ & $\begin{array}{l}\text { Open-label, } \\
\text { multicenter } \\
\text { randomized } \\
\text { controlled } \\
\text { phase } 3\end{array}$ & Docetaxel & 425 & 64 & 61 & $\begin{array}{l}\text { Docetaxel } 75 \mathrm{mg} / \mathrm{m}^{2} \text {, } \\
\text { on day } 1 \text {, every } \\
3 \text { weeks }\end{array}$ & 160 & 165 & & 315 & 110 \\
\hline $\begin{array}{l}\text { Butts } \\
\text { et al (27) } \\
2014\end{array}$ & $\begin{array}{l}\text { START } \\
\text { randomized, } \\
\text { double-blind } \\
\text { multicenter, } \\
\text { phase } 3\end{array}$ & Placebo & 410 & 61.5 & 68 & $\begin{array}{l}\text { Liposome } \\
\text { forming lipids }\end{array}$ & 167 & 239 & & 163 & 171 \\
\hline $\begin{array}{l}\text { Herbst } \\
\text { et al (28) } \\
2011\end{array}$ & $\begin{array}{l}\text { Double-blind, } \\
\text { multicenter, } \\
\text { placebo-controlled, } \\
\text { phase } 3 \text { trial }\end{array}$ & $\begin{array}{l}\text { Erlotinib } \\
\text { (EGFR inhibitor) }\end{array}$ & 317 & 64.8 & 54 & $\begin{array}{l}\text { Erlotinib } \\
150 \mathrm{mg} / \text { day }\end{array}$ & 121 & 176 & 20 & 90 & 17 \\
\hline $\begin{array}{l}\text { Giaccone } \\
\text { et al (29) } \\
2015\end{array}$ & Phase 3 study & Placebo & 262 & 60.5 & 58 & $0.15 \%$ intralipid & 130 & 119 & 6 & 141 & 81 \\
\hline
\end{tabular}

ECOG status, Eastern Cooperative Oncology Group performance status; Ad, adenocarcinoma; Sq, squamous cell carcinoma; pfu, plaque forming units; cisp, cisplatin; gem, gemcitabine; carbo, carboplatin; pacli, paclitaxel; Mab, monoclonal antibody.

lipids (27). Belagenpumatucel-L was given intradermally at a dose of $2.5 \times 10^{7}$ total cells (29). Chemotherapy generally included carboplatin, paclitaxel, docetaxel, cisplatin and gemcitabine. In one study (28), EGFR inhibitor, erlotinib was used as chemotherapy agent in control group patients. Where chemotherapy was not the regimen for control group patients, vehicle (used for immunotherapy) placebo (intralipid or liposome forming lipids) was given.

Effect of immunotherapy on PFS and OS. In pooled OR analysis (Mantel-Haenszel, random) of both types of immunotherapies, PFS was found to be significantly increased (Fig. 2A) in a higher number of patients as compared to chemotherapy/placebo control group (OR 1.81, 95\% CI 1.36, 2.42; $\mathrm{P}<0.0001)$. Further separate analysis of antibody therapy also revealed a significant beneficial effect on PFS in antibody receiving patients (Fig. 2B) as compared to control group (OR 2.05, 95\% CI 1.42, 2.94; P<0.0001). Even though the vaccine therapy-receiving patients also showed better PFS compared to control group (Fig. 2C), the statistical signifi- cance (OR 1.31, 95\% CI 1.05, 1.63; P<0.01) was not as high as in the case of antibody therapy. These results are in accordance with earlier results of a pooled analysis of the beneficial effects of immunotherapies (12). Similar results were obtained by risk ratio (RR) analysis (Table II). Thus, RR for both types of immunotherapies was 1.297 (95\% CI 1.211, 1.389; $\mathrm{P}=0)$ and for Mab therapy RR was 1.335 (95\% CI 1.222, 1.458; $\mathrm{P}=0)$. But RR for vaccine therapy was 1.063 and was not significant (95\% CI 0.96, 1.176; $\mathrm{P}=0.239$ ).

OS was significantly enhanced by both the immunotherapies as compared to chemotherapy/placebo controls (Fig. 3A), as revealed by mean difference analysis in IV fixed mode $(\mathrm{P}<0.00001)$. Unlike in the case of PFS, OS was greatly improved by both antibody-based therapy (Fig. 3B; $\mathrm{P}<0.00001$ ) and vaccine-based therapy (Fig. $3 \mathrm{C} ; \mathrm{P}<0.00001$ ). These results are in agreement with earlier findings showing the beneficial effects of immunotherapies on OS $(12,16)$.

Effect of immunotherapies on treatment related adverse effects. Earlier studies indicated that hematological adverse 


\section{A Both immunotherapies}

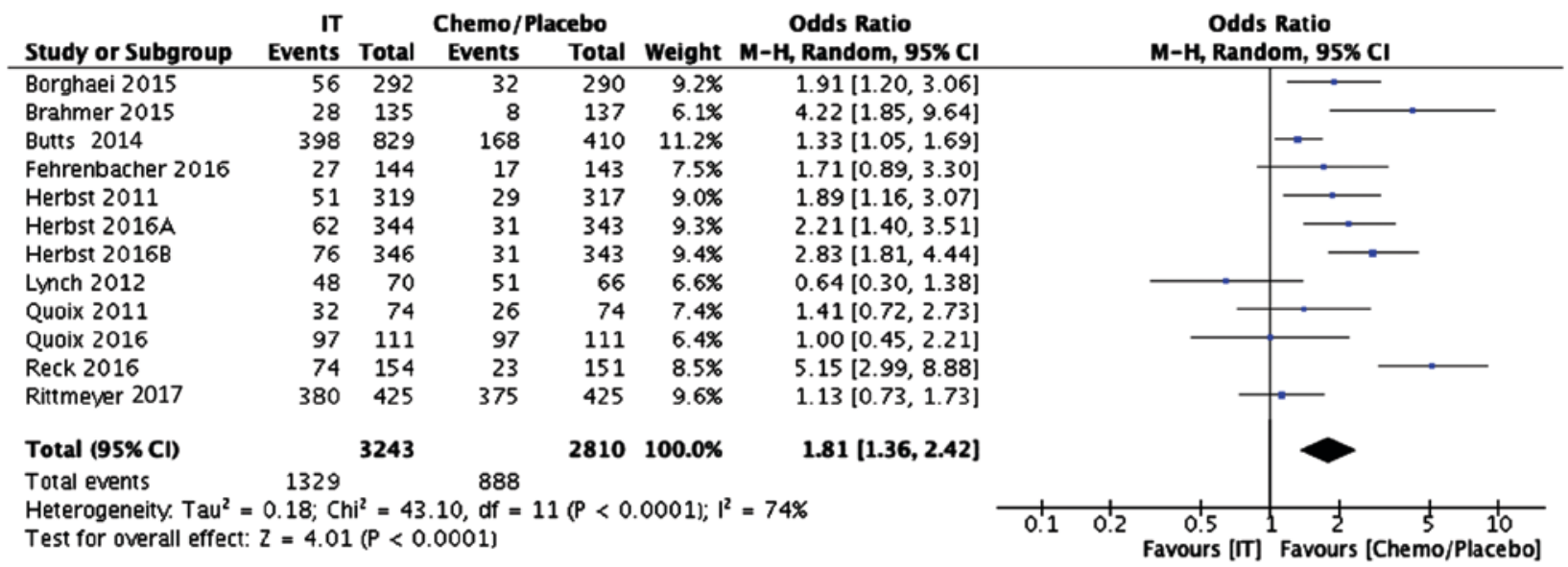

\section{B Antibody immunotherapy}

\begin{tabular}{|c|c|c|c|c|c|c|c|c|c|}
\hline Study or Subgroup & \multicolumn{2}{|c|}{ IT-Ab } & \multicolumn{2}{|c|}{ Chemo/Placebo } & \multicolumn{3}{|c|}{ Odds Ratio } & \multicolumn{2}{|c|}{$\begin{array}{c}\text { Odds Ratio } \\
\text { M-H, Random, 95\% Cl }\end{array}$} \\
\hline Herbst 2011 & 51 & 319 & 29 & 317 & $11.9 \%$ & $1.89[1.16,3.07]$ & 2011 & & \\
\hline Lynch 2012 & 48 & 70 & 51 & 66 & $9.1 \%$ & $0.64[0.30,1.38]$ & 2012 & & 一 \\
\hline Borghaei 2015 & 56 & 292 & 32 & 290 & $12.1 \%$ & $1.91[1.20,3.06]$ & 2015 & & \\
\hline Brahmer 2015 & 28 & 135 & 8 & 137 & $8.5 \%$ & $4.22[1.85,9.64]$ & 2015 & & \\
\hline Rittmeyer 2017 & 380 & 425 & 375 & 425 & $12.5 \%$ & $1.13[0.73,1.73]$ & 2016 & & \\
\hline Reck 2016 & 74 & 154 & 23 & 151 & $11.3 \%$ & $5.15[2.99,8.88]$ & 2016 & & \\
\hline Herbst 20168 & 76 & 346 & 31 & 343 & $12.3 \%$ & $2.83[1.81,4.44]$ & 2016 & & \\
\hline Herbst 2016A & 62 & 344 & 31 & 343 & $12.2 \%$ & $2.21[1.40,3.51]$ & 2016 & & \\
\hline Fehrenbacher 2016 & 27 & 144 & 17 & 143 & $10.1 \%$ & $1.71[0.89,3.30]$ & 2016 & & \\
\hline Total (95\% Cl) & & 2229 & & 2215 & $100.0 \%$ & $2.05[1.42,2.94]$ & & & \\
\hline Total events & 802 & & 597 & & & & & & \\
\hline $\begin{array}{l}\text { Heterogeneity. Tau }{ }^{2} \\
\text { Test for overall effect }\end{array}$ & $\begin{array}{l}23 ; \mathrm{Ch} \\
=3.86\end{array}$ & $=$ & $\begin{array}{l}.90, \mathrm{df}= \\
.00011\end{array}$ & 0 & $001)$ & $76 \%$ & & $\begin{array}{ccc}0.1 & 0.2 & 0.5 \\
& \text { Favours [IT-Ab] }\end{array}$ & Favours \\
\hline
\end{tabular}

\section{Vaccine-based immunotherapy}

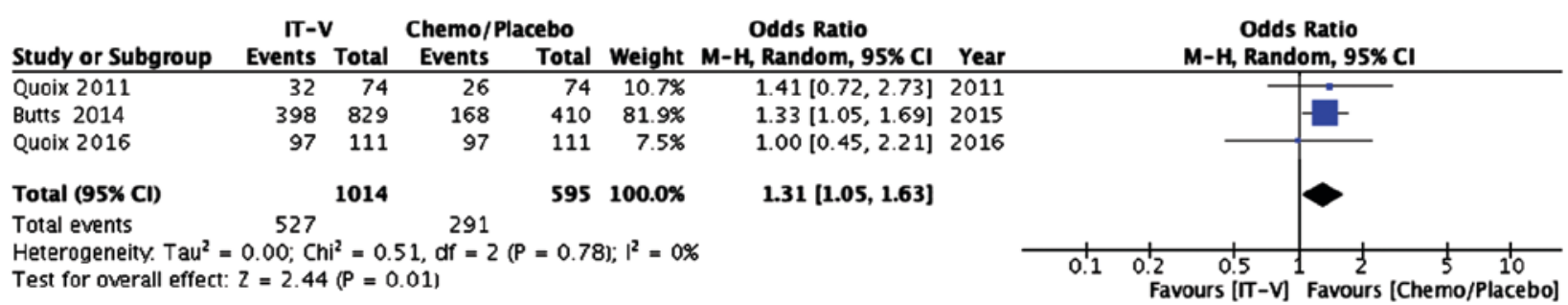

Figure 2. Effect of immunotherapies on progression-free survival in NSCLC patients, compared to chemotherapy or placebo. Comparison of chemotherapy/ placebo with both the (A) immunotherapies, (B) antibody therapy and (C) vaccine-based therapy. Forest plots of Odds ratio, analyzed by Mantel-Haenszel statistics in the random-effect model. NSCLC, non-small cell lung cancer.

Table II. Risk ratio analysis of PFS in antibody-based and vaccine-based immunotherapy groups vs. corresponding chemotherapy/placebo groups.

\begin{tabular}{|c|c|c|c|c|c|c|c|c|c|c|}
\hline \multirow[b]{2}{*}{ PFS } & \multicolumn{2}{|c|}{ Immunotherapy } & \multicolumn{4}{|c|}{ Chemotherapy/placebo } & \multirow[b]{2}{*}{ Risk ratio } & \multicolumn{2}{|c|}{$95 \% \mathrm{CI}$} & \multirow[b]{2}{*}{ P-value } \\
\hline & Events & Non-events & Total & Events & Non-events & Total & & Lower & $\overline{\text { Upper }}$ & \\
\hline $\begin{array}{l}\text { PFS (for all } \\
\text { immunotherapy) }\end{array}$ & 1,329 & 1,914 & 3,243 & 888 & 1,922 & 2,810 & 1.2968 & 1.2112 & 1.3885 & 0 \\
\hline $\begin{array}{l}\text { PFS (for antibody- } \\
\text { based therapy) }\end{array}$ & 802 & 1,427 & 2,229 & 597 & 1,618 & 2,215 & 1.3349 & 1.2223 & 1.4579 & 0 \\
\hline $\begin{array}{l}\text { PFS (for vaccine- } \\
\text { based therapy) }\end{array}$ & 527 & 487 & 1,014 & 291 & 304 & 595 & 1.0627 & 0.9604 & 1.1759 & 0.2392 \\
\hline
\end{tabular}

PFS, progression-free survival; CI, confidence interval. 
A Both immunotherapies

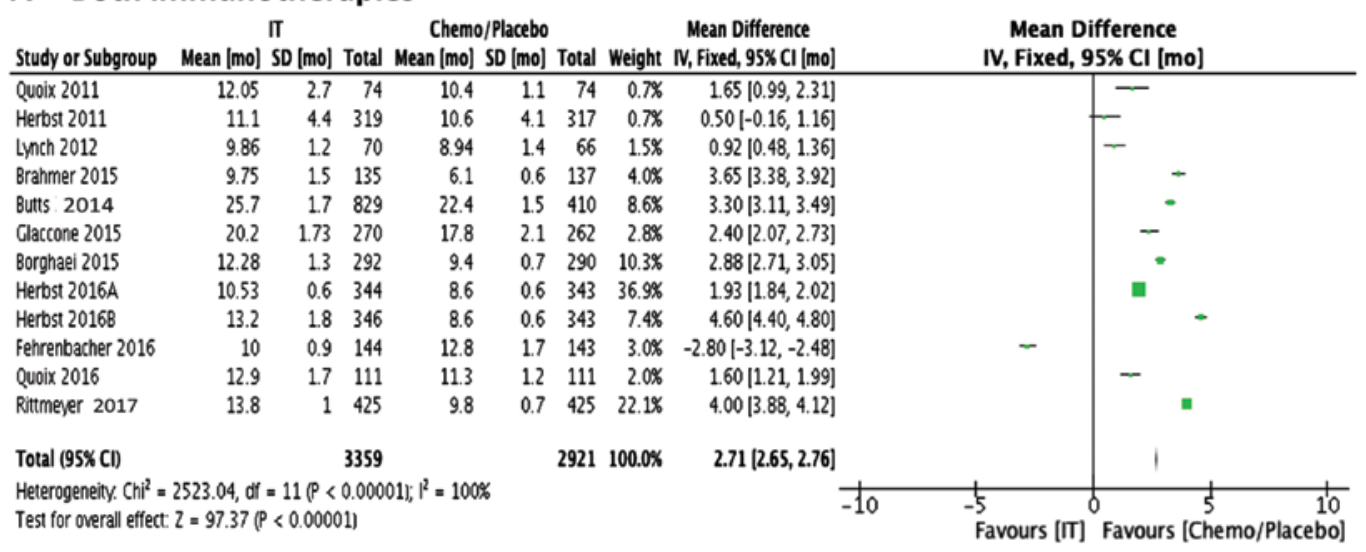

B Antibody immunotherapy

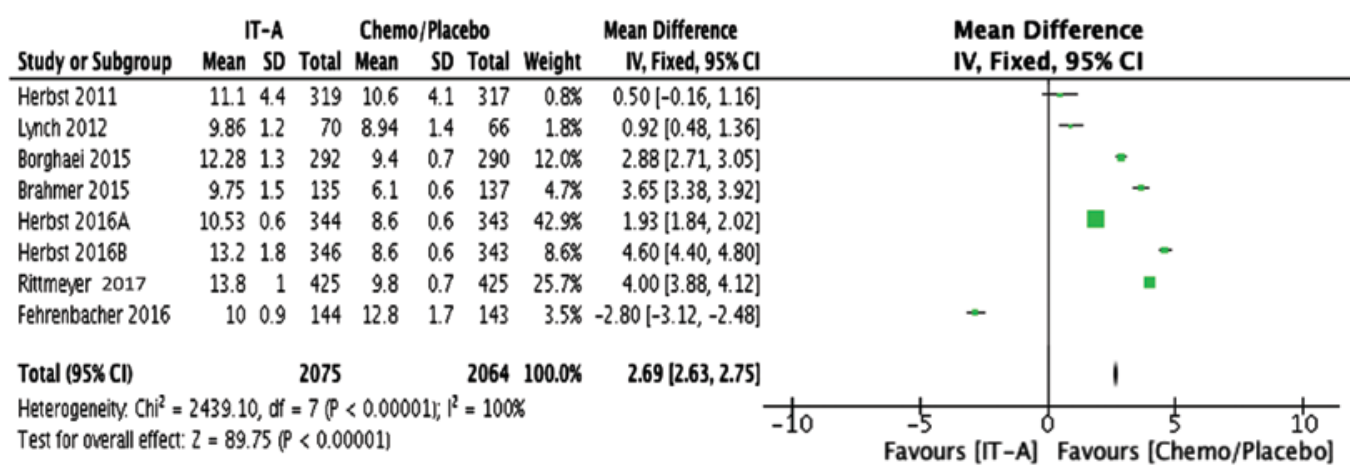

\section{Vaccine-based immunotherapy}

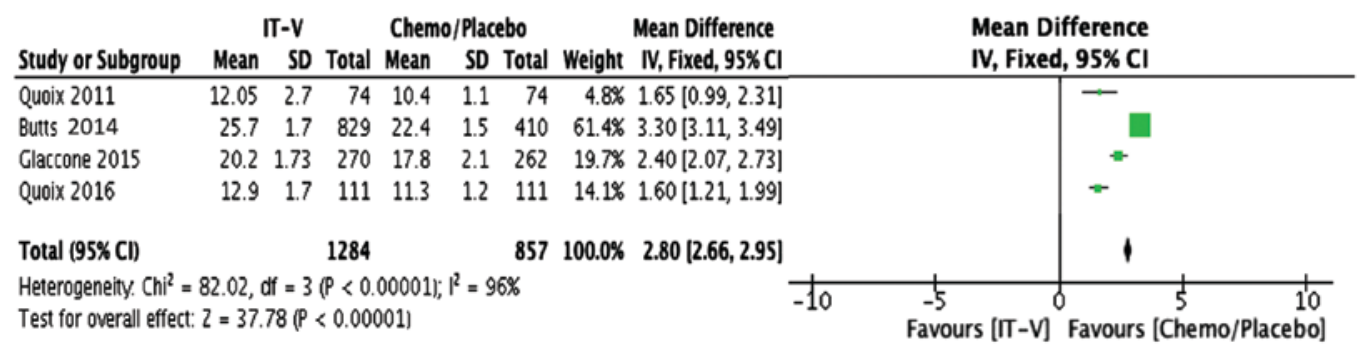

Figure 3. Effect of immunotherapies on overall survival of NSCLC patients, compared to chemotherapy or placebo. Comparison of chemotherapy/placebo with both the (A) immunotherapies, (B) antibody therapy and (C) vaccine-based therapy. Forest plots of mean difference analyzed by IV analysis in fixed-effect model. NSCLC, non-small cell lung cancer; IV, inverse variance.

effects were relatively lower in patients treated with immune check point inhibitors whereas in vaccine treated patients these effects were observed often (16). In the present meta-analysis (OR, Mantel-Haenszel, random), we observed that among the hematological adverse effects (Fig. 4), anemia (Fig. 4A; OR 0.27, 95\% CI 0.15, 0.49; P<0.0001) and neutropenia (Fig. 4B; OR 0.09, 95\% CI 0.02, 0.35; P=0.0004) are significantly lower in the immunotherapy-receiving patients (Table III). However, events of thrombocytopenia (Fig. 4C; OR $0.68,95 \%$ CI $0.31,1.51 ; \mathrm{P}=0.34$ ) are not different compared to chemotherapy/placebo control group. Thrombocytopenia events were reported only in approximately half of the included studies, unlike anemia and neutropenia. It has been shown earlier that thrombocytopenia events are similar or even more common in patients treated with immunomodulatory therapy (16). Among the non-hematological adverse effects, neither abdominal pain nor loss of appetite/anorexia was found to be different in the immunotherapy group (Fig. 5A and B). However, fatigue, which is a commonly seen adverse effect appeared to be much less common in the immunotherapy group, as compared to the control chemotherapy/placebo group (Fig. 5C; OR 0.67, 95\% CI 0.52, 0.86; P<0.001). A closer look at the data indicated that the number of patients with fatigue are much less in the antibody treated group, compared to their controls, whereas the events seems to be higher in the vaccine treated group. Other adverse effects including nausea, fever and vomiting are not much different in the immunotherapy group (Fig. 6).

Sensitivity analysis. In order to assess the robustness and to eliminate bias in the results, we re-analyzed the PFS and OS data by excluding individual trials with highest or lowest 


\section{A Anemia}

IT Chemo/Placebo Odds Ratio

\begin{tabular}{|c|c|c|c|c|c|c|c|c|}
\hline Study or Subgroup & Events & Total & $\begin{array}{l}\text { Cnemo//R } \\
\text { Events }\end{array}$ & Total & Weight & $\mathrm{M}-\mathrm{H}$, Random, $95 \% \mathrm{Cl}$ & M-H, Ran & dom, $95 \% \mathrm{Cl}$ \\
\hline Quoix 2011 & 32 & 74 & 43 & 74 & $10.8 \%$ & $0.55[0.29,1.05]$ & $\rightarrow$ & \\
\hline Lynch 2012 & 65 & 71 & 62 & 65 & $7.2 \%$ & $0.52[0.13,2.19]$ & & \\
\hline Brahmer 2015 & 2 & 135 & 28 & 137 & $7.1 \%$ & $0.06[0.01,0.25]$ & & \\
\hline Borghaei 2015 & 6 & 287 & 53 & 268 & $9.9 \%$ & $0.09[0.04,0.21]$ & & \\
\hline Rittmeyer 2017 & 70 & 609 & 136 & 578 & $12.0 \%$ & $0.42[0.31,0.58]$ & $\rightarrow$ & \\
\hline Reck 2016 & 8 & 154 & 66 & 151 & $10.2 \%$ & $0.07[0.03,0.15]$ & & \\
\hline Quoix 2016 & 55 & 110 & 43 & 107 & $11.3 \%$ & $1.49[0.87,2.55]$ & & $\rightarrow$ \\
\hline Herbst 2016B & 14 & 343 & 40 & 309 & $10.9 \%$ & $0.29[0.15,0.54]$ & & \\
\hline Herbst 2016A & 10 & 339 & 40 & 309 & $10.6 \%$ & $0.20[0.10,0.42]$ & & \\
\hline Fehrenbacher 2016 & 8 & 142 & 22 & 135 & $9.9 \%$ & $0.31[0.13,0.72]$ & & \\
\hline Total $(95 \% \mathrm{Cl})$ & & 2264 & & 2133 & $100.0 \%$ & $0.27[0.15,0.49]$ & & \\
\hline Total events & 270 & & 533 & & & & & \\
\hline $\begin{array}{l}\text { Heterogeneity. Tau }{ }^{2}= \\
\text { Test for overall effect: }\end{array}$ & $\begin{array}{l}0.74 ; C h \\
z=4.31\end{array}$ & $\begin{array}{l}h^{2}=6 \\
1(P<C\end{array}$ & $\begin{array}{l}04, d f=9 \\
0001]\end{array}$ & $<0.0$ & בחי & $7 \%$ & $\begin{array}{l}0.1 \\
\text { Favours ! }\end{array}$ & 1 Favours \\
\hline
\end{tabular}

\section{B Neutropenia}

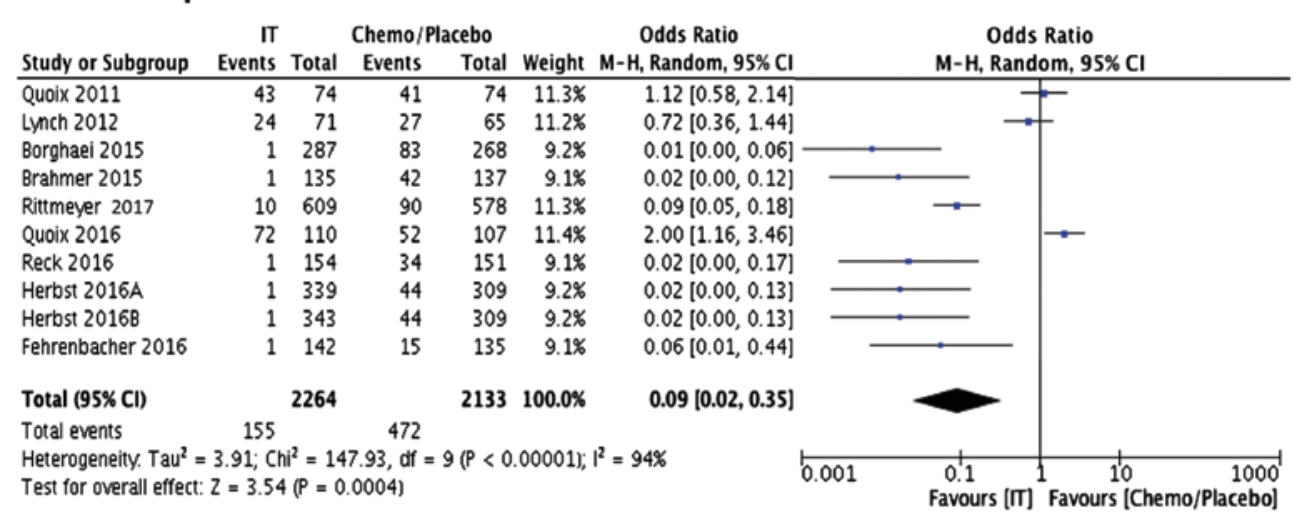

\section{Thrombocytopenia}

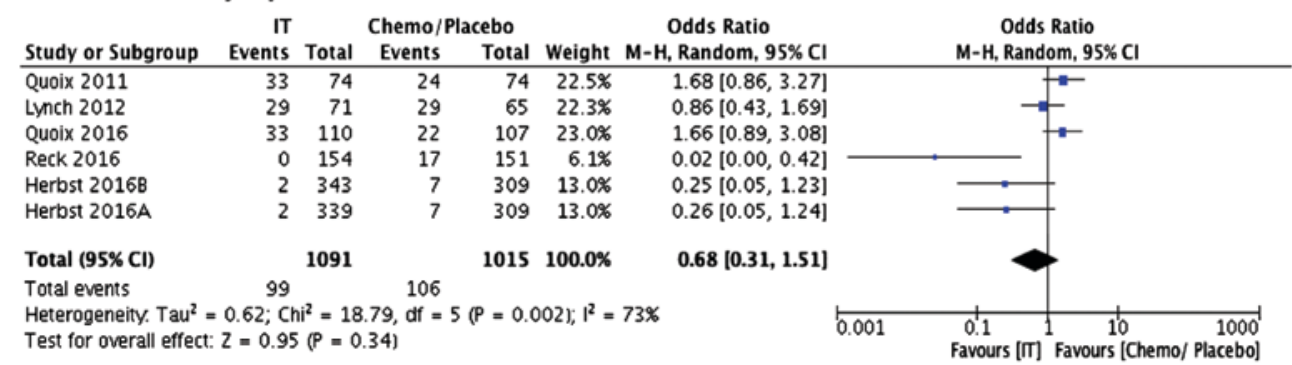

Figure 4. Effect of immunotherapies on adverse hematological effects. Comparison of chemotherapy/placebo with both the immunotherapies on the events of (A) anemia, (B) neutropenia and (C) thrombocytopenia. Forest plots of Odds ratio, analyzed by Mantel-Haenszel statistics in the random-effect model.

Table III. Risk ratio analysis of adverse effects in immunotherapy vs. chemotherapy/placebo groups.

\begin{tabular}{|c|c|c|c|c|c|c|c|c|c|c|}
\hline \multirow[b]{2}{*}{ Event measured } & \multicolumn{3}{|c|}{ Immunotherapy } & \multicolumn{3}{|c|}{ Chemotherapy/placebo } & \multirow[b]{2}{*}{ Risk ratio } & \multicolumn{2}{|c|}{$95 \% \mathrm{CI}$} & \multirow[b]{2}{*}{ P-value } \\
\hline & Events & Non-events & Total & Events & Non-events & Total & & Lower & Upper & \\
\hline Anemia & 270 & 1,994 & 2,264 & 533 & 1,600 & 2,133 & 0.4773 & 0.4174 & 0.5457 & 0 \\
\hline Neutropenia & 155 & 2,109 & 2,264 & 472 & 1,661 & 2,133 & 0.3094 & 0.2606 & 0.3673 & 0 \\
\hline Thrombocytopenia & 99 & 992 & 1,091 & 106 & 909 & 1,015 & 0.8689 & 0.6698 & 1.1272 & 0.2899 \\
\hline Abdominal pain & 51 & 1,288 & 1,339 & 45 & 1,159 & 1,204 & 1.0191 & 0.6877 & 1.51 & 0.925 \\
\hline Nausea & 544 & 3,014 & 3,558 & 598 & 2,274 & 2,872 & 0.7343 & 0.661 & 0.8158 & 0 \\
\hline Fatigue & 766 & 2,792 & 3,558 & 841 & 2,031 & 2,872 & 0.7352 & 0.6755 & 0.8002 & 0 \\
\hline Vomiting & 206 & 2,058 & 2,264 & 265 & 1,868 & 2,133 & 0.7324 & 0.6165 & 0.87 & 0.0004 \\
\hline Anorexia & 553 & 2,934 & 3,487 & 486 & 2,321 & 2,807 & 0.916 & 0.8195 & 1.0238 & 0.1221 \\
\hline Fever & 230 & 2,123 & 2,353 & 184 & 2,039 & 2,223 & 1.1809 & 0.9815 & 1.4209 & 0.0781 \\
\hline
\end{tabular}

CI, confidence interval. 
A Abdominal pain

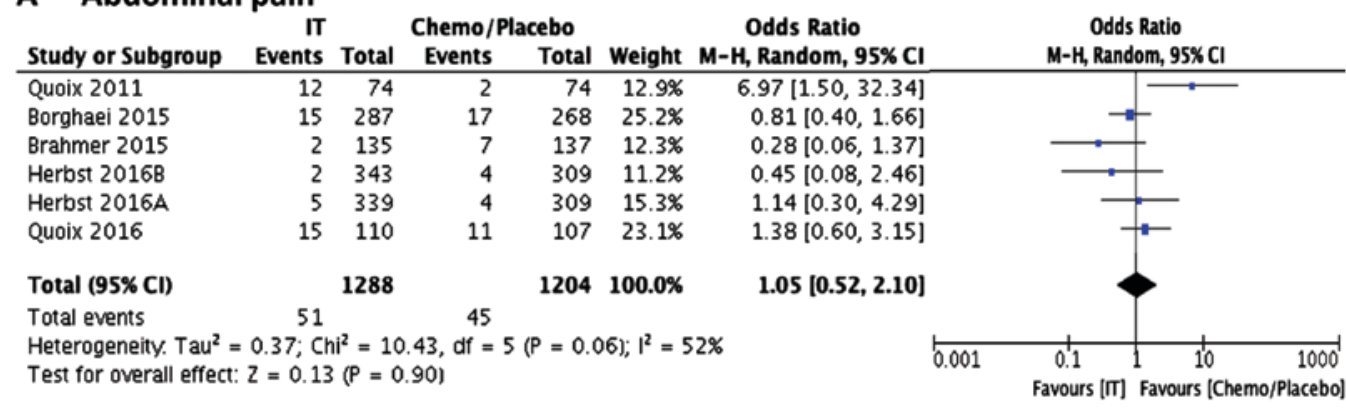

\section{B Anorexia/ Loss of appetite}

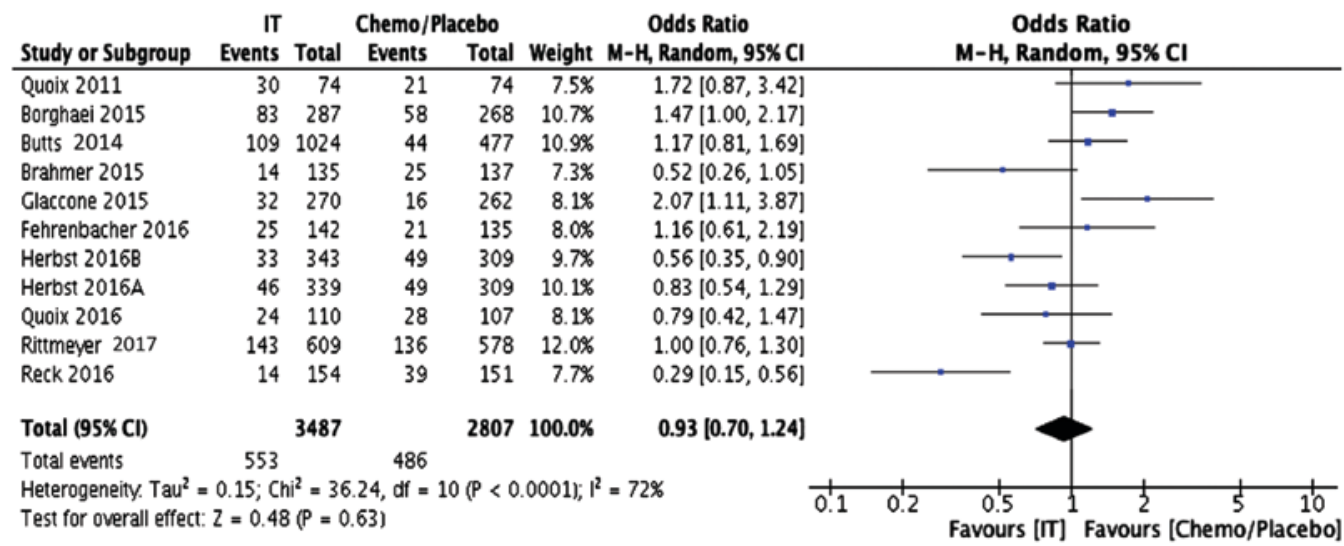

\section{Fatigue}

\begin{tabular}{|c|c|c|c|c|c|c|c|c|}
\hline Study or Subgroup & \multicolumn{2}{|l|}{ IT } & \multicolumn{2}{|c|}{ Chemo/Placebo } & Weight & \multirow{2}{*}{$\begin{array}{c}\begin{array}{c}\text { Odds Ratio } \\
\text { M-H, Random, 95\% Cl }\end{array} \\
1.47[0.77,2.81]]\end{array}$} & \multicolumn{2}{|c|}{$\begin{array}{c}\text { Odds Ratio } \\
\text { M-H, Random, } 95 \% \mathrm{Cl}\end{array}$} \\
\hline Quoix 2011 & 44 & 74 & 37 & 74 & $6.6 \%$ & & - & \\
\hline Lynch 2012 & 20 & 71 & 18 & 65 & $5.7 \%$ & $1.02[0.48,2.17]$ & & \\
\hline Butts 2014 & 197 & 1024 & 102 & 477 & $10.7 \%$ & $0.88[0.67,1.15]$ & & + \\
\hline Brahmer 2015 & 21 & 135 & 42 & 137 & $7.2 \%$ & $0.42[0.23,0.75]$ & & \\
\hline Borghaei 2015 & 46 & 287 & 78 & 268 & $9.1 \%$ & $0.46[0.31,0.70]$ & & \\
\hline Glaccone 2015 & 66 & 270 & 54 & 262 & $9.2 \%$ & $1.25[0.83,1.87]$ & & \\
\hline Quoix 2016 & 69 & 110 & 63 & 107 & $7.6 \%$ & $1.18[0.68,2.03]$ & & \\
\hline Reck 2016 & 16 & 154 & 43 & 151 & $6.8 \%$ & $0.29[0.16,0.54]$ & & \\
\hline Rittmeyer 2017 & 163 & 609 & 205 & 578 & $10.9 \%$ & $0.66[0.52,0.85]$ & & \\
\hline Herbst 2016B & 49 & 343 & 76 & 309 & $9.3 \%$ & $0.51[0.34,0.76]$ & & \\
\hline Herbst 2016A & 46 & 339 & 76 & 309 & $9.2 \%$ & $0.48[0.32,0.72]$ & & \\
\hline Fehrenbacher 2016 & 29 & 142 & 47 & 135 & $7.7 \%$ & $0.48[0.28,0.82]$ & & \\
\hline Total $(95 \% \mathrm{Cl})$ & & 3558 & & 2872 & $100.0 \%$ & $0.67[0.52,0.86]$ & & \\
\hline Total events & 766 & & 841 & & & & & \\
\hline $\begin{array}{l}\text { Heterogeneity. Tau² = } \\
\text { Test for overall effect: }\end{array}$ & $\begin{array}{l}0.13 ; C h \\
z=3.20\end{array}$ & $r^{*}=$ & 1.61, df $=1$ & $P<$ & Do1); & $74 \%$ & $\begin{array}{ccc}1 & 1 & 1 \\
0.1 & 0.2 & 0.5 \\
& & \text { Favours [IT] }\end{array}$ & $1 \frac{1}{2}$ \\
\hline
\end{tabular}

Figure 5. Effect of immunotherapies on adverse non-hematological effects. Comparison of chemotherapy/placebo with both the immunotherapies on the events of (A) abdominal pain, (B) anorexia/loss of appetite and (C) fatigue. Forest plots of Odds ratio, analyzed by Mantel-Haenszel statistics in the random-effect model.

weightage. Such analysis did not qualitatively alter the obtained results and conclusions (data not elaborated). The OS benefits were still noted by immunotherapy over the chemotherapy/placebo controls. These conclusions are in agreement with earlier study (16), indicating that the benefits of immunotherapy are real and reproducible.

\section{Discussion}

Chemotherapy and chemoradiotherapy are the first-line therapy for several cancers including NSCLC. Even though objective responses have been noted with many of these therapies, the efficacy is not observed in all the patients. Tyrosine kinase receptor inhibitors showed better effects on
OS and PFS but due to resistance, their efficacy is lost and disease progression takes place. A better understanding of immune mechanisms and host antitumor responses led to the identification of potential therapeutic opportunities employing the components of immune system. These include different types of immunotherapies based on the use of monoclonal antibodies against tumor specific antigens and immune checkpoint pathways, immunomodulators, vaccination against tumor antigens and activated immune cells that attack tumors $(8,30)$. In the present meta-analysis, we examined the effectiveness of immunotherapies in improving PFS and OS. A total of 13 multicenter international phase II and III clinical trials addressing the efficacy of antibody therapies and vaccine therapies for treating NSCLC patients are included in this 


\begin{tabular}{|c|c|c|c|c|c|c|c|c|}
\hline $\begin{array}{l}\text { A Nausea } \\
\text { Study or Subgroup }\end{array}$ & $\begin{array}{r}\text { IT } \\
\text { Events }\end{array}$ & Total & $\begin{array}{l}\text { Chemo/Pla } \\
\text { Events }\end{array}$ & $\begin{array}{l}\text { acebo } \\
\text { Total }\end{array}$ & Weight & $\begin{array}{c}\text { Odds Ratio } \\
\text { M-H, Random, } 95 \% \mathrm{CI}\end{array}$ & $\begin{array}{r}\text { Odds } \\
\text { M-H, Rand }\end{array}$ & $\begin{array}{l}\text { Ratio } \\
\text { lom, } 95 \% \mathrm{Cl}\end{array}$ \\
\hline Quoix 2011 & 36 & 74 & 32 & 74 & $7.8 \%$ & $1.24[0.65,2.38]$ & & 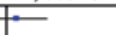 \\
\hline Lynch 2012 & 19 & 71 & 21 & 65 & $7.3 \%$ & $0.77[0.37,1.60]$ & $\longrightarrow$ & - \\
\hline Borghaei 2015 & 34 & 287 & 70 & 268 & $8.8 \%$ & $0.38[0.24,0.60]$ & - & \\
\hline Glaccone 2015 & 40 & 270 & 36 & 262 & $8.6 \%$ & $1.09[0.67,1.78]$ & & - \\
\hline Brahmer 2015 & 12 & 135 & 30 & 137 & $7.4 \%$ & $0.35[0.17,0.71]$ & — & \\
\hline Butts 2014 & 140 & 1024 & 39 & 477 & $9.2 \%$ & $1.78[1.23,2.58]$ & & $\rightarrow$ \\
\hline Herbst 20168 & 31 & 343 & 45 & 309 & $8.6 \%$ & $0.58[0.36,0.95]$ & .ـ. & \\
\hline Herbst 2016A & 37 & 339 & 45 & 309 & $8.7 \%$ & $0.72[0.45,1.14]$ & & \\
\hline Rittmeyer 2017 & 108 & 609 & 131 & 578 & $9.5 \%$ & $0.74[0.55,0.98]$ & & \\
\hline Reck 2016 & 15 & 154 & 65 & 151 & $7.9 \%$ & $0.14[0.08,0.27]$ & & \\
\hline Quoix 2016 & 55 & 110 & 47 & 107 & $8.4 \%$ & $1.28[0.75,2.18]$ & & - \\
\hline Fehrenbacher 2016 & 17 & 142 & 37 & 135 & $7.8 \%$ & $0.36[0.19,0.68]$ & & \\
\hline Total $(95 \% \mathrm{Cl})$ & & 3558 & & 2872 & $100.0 \%$ & $0.65[0.45,0.95]$ & & \\
\hline & 544 & & 598 & & & & & \\
\hline $\begin{array}{l}\text { Heterogeneity. Taú, } \\
\text { Test for overall effect }\end{array}$ & $\begin{array}{l}36 ; \mathrm{Chi} \\
=2.21\end{array}$ & $(P=$ & $\begin{array}{l}.57, \text { df }= \\
.03)\end{array}$ & $(P<$ & $00001)$ & $85 \%$ & $\begin{array}{l}0.1 \\
\text { Favours [I] }\end{array}$ & Favours $|C|$ \\
\hline
\end{tabular}

\section{B Fever}

\begin{tabular}{|c|c|c|c|c|c|c|c|c|c|c|}
\hline Study or Subgroup & \multicolumn{2}{|l|}{ IT } & \multicolumn{2}{|c|}{ Chemo/Placebo } & Weight & $\begin{array}{c}\text { Odds Ratio } \\
\mathrm{M}-\mathrm{H} \text {, Random, } 95 \% \mathrm{Cl}\end{array}$ & \multicolumn{4}{|c|}{$\begin{array}{c}\text { Odds Ratio } \\
\mathrm{M}-\mathrm{H} \text {, Random, } 95 \% \mathrm{Cl}\end{array}$} \\
\hline Quoix 2011 & 17 & 74 & 6 & 74 & $8.5 \%$ & $3.38[1.25,9.14]$ & & & & \\
\hline Glaccone 2015 & 25 & 270 & 17 & 262 & $12.6 \%$ & $1.47[0.77,2.79]$ & & & & \\
\hline Borghaei 2015 & 8 & 287 & 17 & 268 & $9.9 \%$ & $0.42[0.18,1.00]$ & & & & \\
\hline Brahmer 2015 & 6 & 135 & 10 & 137 & $8.1 \%$ & $0.59[0.21,1.67]$ & & & & \\
\hline Reck 2016 & 16 & 154 & 8 & 151 & $9.7 \%$ & $2.07[0.86,5.00]$ & & & & \\
\hline Herbst 2016A & 10 & 339 & 17 & 309 & $10.6 \%$ & $0.52[0.24,1.16]$ & & & & \\
\hline Herbst 2016B & 14 & 343 & 17 & 309 & $11.5 \%$ & $0.73[0.35,1.51]$ & & & & \\
\hline Fehrenbacher 2016 & 26 & 142 & 16 & 135 & $12.2 \%$ & $1.67[0.85,3.27]$ & & & & \\
\hline Rittmeyer 2017 & 108 & 609 & 76 & 578 & $17.0 \%$ & $1.42[1.04,1.96]$ & & & & \\
\hline Total $(95 \% \mathrm{Cl})$ & & 2353 & & 2223 & $100.0 \%$ & $1.12[0.76,1.65]$ & & & & \\
\hline Total events & 230 & & 184 & & & & & & & \\
\hline $\begin{array}{l}\text { Heterogeneity. Tau }{ }^{2} \\
\text { Test for overall effect }\end{array}$ & $\begin{array}{l}0.20 ; C h \\
Z=0.57\end{array}$ & $\begin{array}{l}i^{2}=2 \\
(P=\end{array}$ & $\begin{array}{l}47, \mathrm{df}=8 \\
57)\end{array}$ & $=$ & 01,1 & & $\begin{array}{cc}1 & 1 \\
0.1 & 0.2\end{array}$ & $\begin{array}{c}0.5 \\
\text { Favours [IT] }\end{array}$ & Favours [C & $\stackrel{5}{5}_{\text {mo/Placebo] }}^{10}$ \\
\hline
\end{tabular}

\section{Vomiting}

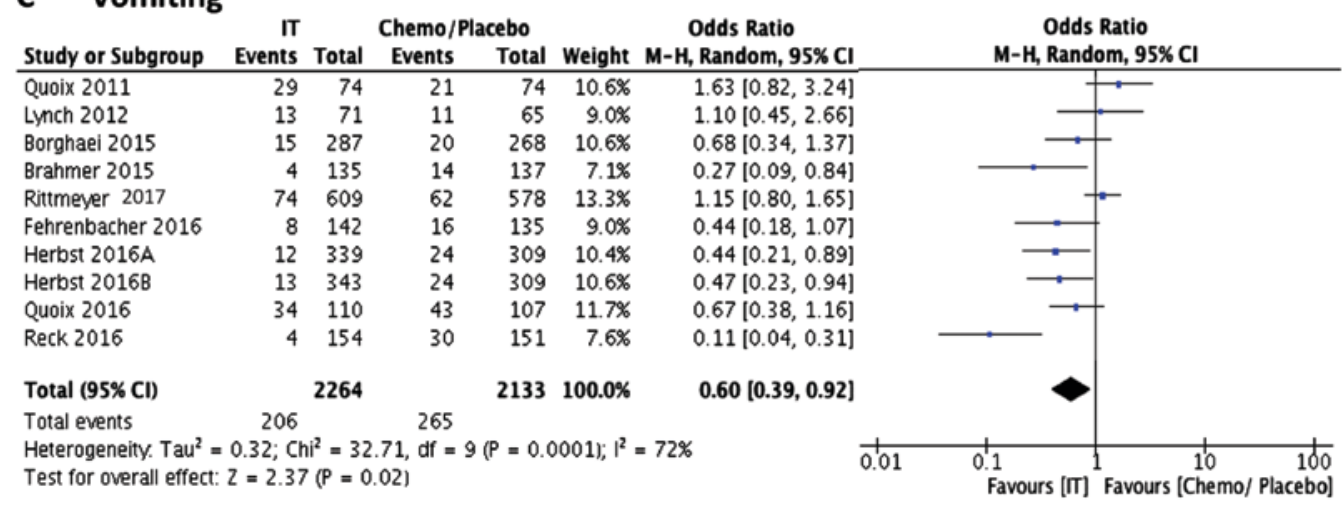

Figure 6. Effect of immunotherapies on nausea, fever and vomiting. Comparison of chemotherapy/placebo with both the immunotherapies on the events of (A) nausea, (B) fever and (C) vomiting. Forest plots of Odds ratio, analyzed by Mantel-Haenszel statistics in the random-effect model.

analysis. Among these studies, 9 were Mab-based therapies targeting different antigens and 4 were vaccine-based, with different immunogens. The immune check point pathway targets of the employed Mabs were programmed death-1 (PD1), programmed death ligand-1 (PD-L1) and cytotoxic T-lymphocyte-associated antigen-4 (CTLA-4). Vaccine therapies were targeting the MUC1 tumor-associated antigen and using NSCLC cells transfected with human transforming growth factor- $\beta 2$-antisense vector.

In line with other studies, we observed that immunotherapies offer better efficacy in terms of improved PFS and OS. Surprisingly, we observed that Mab therapies are more effective in improving PFS than the vaccine-based therapies $(25,26,28)$. This can be because of the less number of vaccine-based studies and less number of patients in this analysis. An earlier meta-analysis indicated that immunotherapies have relatively stronger effect in improving PFS in low-stage NSCLC than in high-stage NSCLC patients (12). It was also observed that tumor histology is not related to disease progression. Combination of chemotherapy with immunotherapy has been proposed to have synergistic effect in inducing tumor cell death and by disrupting the immune evasion pathways of the tumor cells (31). Blockade of PD-1/PD-L1 signaling with checkpoint inhibiting antibodies has been shown to promote antitumor T-cell functionality (32). Efficacy of immunotherapies is likely related to the expression of the corresponding targets in the selected patient population $(33,34)$, which should be considered as a patient selection criterion for such clinical 
trials in future. However, for PD-1/PD-L1 expression this dependence may not always be true $(8,21)$.

Incidence of moderate adverse effects such as anorexia, abdominal pain, nausea, vomiting and fever appeared to be similar in chemotherapy and immunotherapy groups. But incidence of fatigue in immunotherapy receiving patients is less, suggesting an important beneficial effect by this therapy in improving the quality of life of patients. Also, the number of patients with anemia and neutropenia are less in immunotherapy group indicating a less secondary aggravated effect on hematological parameters. It has been recognized that chemotherapy and chemoradiotherapy in the treatment of NSCLC have adverse effects on bone marrow, leading to anemia, neutropenia and also thrombocytopenia (35). It is possible that immunotherapy assisted antitumor effects are helpful in strengthening the normal cellular homeostatic mechanisms and in overcoming the toxic side effects of chemotherapy. Better hematological parameters are likely further improve the general health and the daily living ability of the patient. Immunotherapy was earlier shown to be safe for agents that trigger specific antitumor reaction $(36,37)$. Thus, overall immunotherapies are safe and well tolerated and when combined with chemotherapy, they are somewhat protective against the toxic effects of chemotherapeutics, particularly on blood parameters. In as much as exercise also has beneficial effects in reducing the risk of lung cancer (5), it is of interest to assess whether a combination of immunotherapy with medium level of supervised exercise has any added benefit to the lung cancer patients, who are at 0-1 score for ECOG performance.

A major limitation of the present meta-analysis is the smaller number of included studies in vaccine-based immunotherapy group. Even though we combined both Mab-based therapies and vaccine-based therapies for comparison against chemotherapy controls for getting a larger picture of the effectiveness of immunotherapy, ideal comparison would be separate comparisons for these two types of immunotherapies. This was done for analyzing the effects on PFS and OS, where we noticed specific effects on PFS. Another limitation is that we included one Mab-based therapy study that targeted VEGF (28), instead of a direct tumor specific antigen, we included this study, as VEGF is important for neovascularization of the tumors and for tumor growth and is secreted by tumor cells. Another important limitation is the different treatment durations of the included studies, as this could have influenced the observed PFS and OS. In as much as female patients with NSCLC are known to have better survival and as it has been seen in the present study that in all the included studies there is a higher preponderance of males, it is important to separate males from females when analyzing the beneficiary effects of immunotherapy. This could not be done due to unavailability of individual patient data for all the included studies. Future analyses should take this into consideration to assess if males or females show a different/better response to a given treatment.

In conclusion, immunotherapy of NSCLC is beneficial and shows better efficacy than chemotherapy/placebo in improving PFS and OS. Besides, immunotherapies, due to their less adverse effects, seem to have beneficial impact on the quality of life of patients and the daily living ability.

\section{References}

1. Torre LA, Bray F, Siegel RL, Ferlay J, Lortet-Tieulent J and Jemal A: Global cancer statistics, 2012. CA Cancer J Clin 65: 87-108, 2015.

2. Gordon SB, Bruce NG, Grigg J, Hibberd PL, Kurmi OP, Lam KB, Mortimer K, Asante KP, Balakrishnan K, Balmes J, et al: Respiratory risks from household air pollution in low and middle income countries. Lancet Respir Med 2: 823-860, 2014.

3. Haghgoo SM, Allameh A, Mortaz E, Garssen J, Folkerts G, Barnes PJ and Adcock IM: Pharmacogenomics and targeted therapy of cancer: Focusing on non-small cell lung cancer. Eur J Pharmacol 754: 82-91, 2015.

4. Madureira P, de Mello RA, de Vasconcelos A and Zhang Y: Immunotherapy for lung cancer: For whom the bell tolls? Tumour Biol 36: 1411-1422, 2015.

5. Sun JY, Shi L, Gao XD and Xu SF: Physical activity and risk of lung cancer: A meta-analysis of prospective cohort studies. Asian Pac J Cancer Prev 13: 3143-3147, 2012.

6. Wang J, Zou ZH, Xia HL, He JX, Zhong NS and Tao AL: Strengths and weaknesses of immunotherapy for advanced non-small-cell lung cancer: A meta-analysis of 12 randomized controlled trials. PLoS One 7: e32695, 2012.

7. Jin ZY, Zhao XT, Zhang LN, Wang Y, Yue WT and Xu SF: Effects of polymorphisms in the XRCC1, XRCC3, and XPG genes on clinical outcomes of platinum-based chemotherapy for treatment of non-small cell lung cancer. Genet Mol Res 13: 7617-7625, 2014.

8. Gridelli C, Ascierto PA, Barberis MC, Felip E, Garon EB, O'Brien M, Senan S, Casaluce F, Sgambato A, Papadimitrakopoulou V and De Marinis F: Immunotherapy of non-small cell lung cancer: Report from an international experts panel meeting of the Italian Association of Thoracic Oncology. Expert Opin Biol Ther 16: 1479-1489, 2016.

9. Forde PM, Kelly RJ and Brahmer JR: New strategies in lung cancer: Translating immunotherapy into clinical practice. Clin Cancer Res 20: 1067-1073, 2014.

10. Kimura H, Matsui Y, Ishikawa A, Nakajima T, Yoshino M and Sakairi Y: Randomized controlled phase III trial of adjuvant chemo-immunotherapy with activated killer $\mathrm{T}$ cells and dendritic cells in patients with resected primary lung cancer. Cancer Immunol Immunother 64: 51-59, 2015.

11. Brahmer J, Reckamp KL, Baas P, Crinò L, Eberhardt WE, Poddubskaya E, Antonia S, Pluzanski A, Vokes EE, Holgado E, et al: Nivolumab versus docetaxel in advanced squamous-cell non-small-cell lung cancer. N Engl J Med 373: 123-135, 2015.

12. Dammeijer F, Lievense LA, Veerman GD, Hoogsteden HC, Hegmans JP, Arends LR and Aerts JG: Efficacy of tumor vaccines and cellular immunotherapies in non-small-cell lung cancer: A systematic review and meta-analysis. J Clin Oncol 34: 3204-3212, 2016.

13. Ramalingam S, Crawford J, Chang A, Manegold C, Perez-Soler R, Douillard JY, Thatcher N, Barlesi F, Owonikoko T, Wang Y, et al: Talactoferrin alfa versus placebo in patients with refractory advanced non-small-cell lung cancer (FORTIS-M trial). Ann Oncol 24: 2875-2880, 2013.

14. Yang L, Shi GL, Song CX and Xu SF: Relationship between genetic polymorphism of MCP-1 and non-small-cell lung cancer in the Han nationality of North China. Genet Mol Res 9: 765-771, 2010.

15. Yang L, Wang LJ, Shi GL, Ni L, Song CX, Zhang ZX and Xu SF: Analysis of HLA-A, HLA-B and HLA-DRB1 alleles in Chinese patients with lung cancer. Genet Mol Res 9: 750-755, 2010.

16. Zhou L, Wang XL, Deng QL, Du YQ and Zhao NQ: The efficacy and safety of immunotherapy in patients with advanced NSCLC: A systematic review and meta-analysis. Sci Rep 6: 32020, 2016.

17. Cuppens K and Vansteenkiste J: Vaccination therapy for non-small-cell lung cancer. Curr Opin Oncol 26: 165-170, 2014.

18. Oken MM, Creech RH, Tormey DC, Horton J, Davis TE, McFadden ET and Carbone PP: Toxicity and response criteria of the Eastern Cooperative Oncology Group. Am J Clin Oncol 5: 649-655, 1982.

19. Quoix E, Ramlau R, Westeel V, Papai Z, Madroszyk A, Riviere A, Koralewski P, Breton JL, Stoelben E, Braun D, et al: Therapeutic vaccination with TG4010 and first-line chemotherapy in advanced non-small-cell lung cancer: A controlled phase 2B trial. Lancet Oncol 12: 1125-1133, 2011.

20. Lynch TJ, Bondarenko I, Luft A, Serwatowski P, Barlesi F, Chacko R, Sebastian M, Neal J, Lu H, Cuillerot JM and Reck M: Ipilimumab in combination with paclitaxel and carboplatin as first-line treatment in stage IIIB/IV non-small-cell lung cancer: Results from a randomized, double-blind, multicenter phase II study. J Clin Oncol 30: 2046-2054, 2012. 
21. Borghaei H, Paz-Ares L, Horn L, Spigel DR, Steins M, Ready NE, Chow LQ, Vokes EE, Felip E, Holgado E, et al: Nivolumab versus docetaxel in advanced nonsquamous non-small-cell lung cancer. N Engl J Med 373: 1627-1639, 2015.

22. Fehrenbacher L, Spira A, Ballinger M, Kowanetz M Vansteenkiste J, Mazieres J, Park K, Smith D, Artal-Cortes A, Lewanski C, et al: Atezolizumab versus docetaxel for patients with previously treated non-small-cell lung cancer (POPLAR): A multicentre, open-label, phase 2 randomised controlled trial. Lancet 387: 1837-1846, 2016.

23. Herbst RS, Baas P, Kim DW, Felip E, Pérez-Gracia JL, Han JY, Molina J, Kim JH, Arvis CD, Ahn MJ, et al: Pembrolizumab versus docetaxel for previously treated, PD-L1-positive, advanced non-small-cell lung cancer (KEYNOTE-010): A randomised controlled trial. Lancet 387: 1540-1550, 2016.

24. Quoix E, Lena H, Losonczy G, Forget F, Chouaid C, Papai Z, Gervais R, Ottensmeier C, Szczesna A, Kazarnowicz A, et al: TG4010 immunotherapy and first-line chemotherapy for advanced non-small-cell lung cancer (TIME): Results from the phase $2 \mathrm{~b}$ part of a randomised, double-blind, placebo-controlled, phase 2b/3 trial. Lancet Oncol 17: 212-223, 2016.

25. Reck M, Rodríguez-Abreu D, Robinson AG, Hui R, Csőszi T, Fülöp A, Gottfried M, Peled N, Tafreshi A, Cuffe S, et al: KEYNOTE-024 Investigators: Pembrolizumab versus chemotherapy for PD-L1-positive non-small-cell lung cancer. N Engl J Med 375: 1823-1833, 2016.

26. Rittmeyer A, Barlesi F, Waterkamp D, Park K, Ciardiello F, von Pawel J, Gadgeel SM, Hida T, Kowalski DM, Dols MC, et al: OAK Study Group: Atezolizumab versus docetaxel in patients with previously treated non-small-cell lung cancer (OAK): A phase 3, open-label, multicentre randomised controlled trial. Lancet 389: 255-265, 2017.

27. Butts C, Socinski MA, Mitchell PL, Thatcher N, Havel L, Krzakowski M, Nawrocki S, Ciuleanu TE, Bosquée L, Trigo JM, et al; START trial team: Tecemotide (L-BLP25) versus placebo after chemoradiotherapy for stage III non-small-cell lung cancer (START): A randomised, double-blind, phase 3 trial. Lancet Oncol 15: 59-68, 2014.
28. Herbst RS, Ansari R, Bustin F, Flynn P, Hart L, Otterson GA, Vlahovic G, Soh CH, O'Connor P and Hainsworth J: Efficacy of bevacizumab plus erlotinib versus erlotinib alone in advanced non-small-cell lung cancer after failure of standard first-line chemotherapy (BeTa): A double-blind, placebo-controlled, phase 3 trial. Lancet 377: 1846-1854, 2011.

29. Giaccone G, Bazhenova LA, Nemunaitis J, Tan M, Juhász E, Ramlau R, van den Heuvel MM, Lal R, Kloecker GH, Eaton KD, et al: A phase III study of belagenpumatucel-L, an allogeneic tumour cell vaccine, as maintenance therapy for non-small cell lung cancer. Eur J Cancer 51: 2321-2329, 2015.

30. Vesely MD, Kershaw MH, Schreiber RD and Smyth MJ: Natural innate and adaptive immunity to cancer. Annu Rev Immunol 29: 235-271, 2011.

31. Emens LA and Middleton G: The interplay of immunotherapy and chemotherapy: Harnessing potential synergies. Cancer Immunol Res 3: 436-443, 2015.

32. Postow MA, Callahan MK and Wolchok JD: Immune checkpoint blockade in cancer therapy. J Clin Oncol 33: 1974-1982, 2015.

33. Noguchi M, Sasada T and Itoh K: Personalized peptide vaccination: A new approach for advanced cancer as therapeutic cancer vaccine. Cancer Immunol Immunother 62: 919-929, 2013.

34. Pol J, Bloy N, Buqué A, Eggermont A, Cremer I, Sautès-Fridman C, Galon J, Tartour E, Zitvogel L, Kroemer G, et al: Trial Watch: Peptide-based anticancer vaccines. Oncoimmunology 4: e974411, 2015.

35. Ettinger DS: Non-small cell lung cancer treatment-related bone marrow toxicities. Semin Oncol 32: S81-S85, 2005.

36. Gelao L, Criscitiello C, Esposito A, Goldhirsch A and Curigliano G: Immune checkpoint blockade in cancer treatment: A double-edged sword cross-targeting the host as an 'innocent bystander'. Toxins (Basel) 6: 914-933, 2014.

37. Weber JS, Yang JC, Atkins MB and Disis ML: Toxicities of immunotherapy for the practitioner. J Clin Oncol 33: 2092-2099, 2015 . 